\title{
A Client-Server Extension to PICSearch system
}

\author{
Kjell Lemström * \\ Department of Computer Science, University of Helsinki \\ Helsinki, Finland
}

\begin{abstract}
The large number of application domains, increased computational power and the possibility to have massive databases have directed many researchers to the area of image retrieval. The remarkable research effort on this particular area has produced a lot of image retrieval algorithms. However, there have not been freely distributed and portable platforms where to easily embed these algorithms. We have made effort to facilitate the work of these researchers by offering a framework system for image retrieval. Earlier, we have introduced a portable, extendible and freely available framework system which can be used with local databases. In this paper we introduce a client-server extension to that PICSearch system. We give an overview to the architectural solution of the client-server system and we present some key ideas of our data communication protocol to minimize the communication rate.
\end{abstract}

\section{Introduction}

The number of different image retrieval algorithms based directly on the features of a query image has drastically increased during the last few years because of the considerable research effort directed to this particular area. A survey of current techniques of image indexing is presented in [7]. However, until these days there have not been any freely available platforms for these kinds of algorithms. The lack of such a systems has led the researchers to create systems on their own just to test their algorithms.

There are various image retrieval systems, such as $Q B I C$ [3], Virage [1], MARS [8] and $I^{2} C$ [10]. The starting point of our project differs from the previous: our primary aim is to help the researchers working in the area of image retrieval by offering a platform where the researchers can easily embed their algorithms. We have created PICSearch [6], which is extendible, portable and freely available (under the GNU General Public License). It also provides an easy-to-use retrieving tool for normal use when several retrieval algorithms have been embedded in it. Furthermore, to our knowledge, our system is the only one that is freely available as a whole.

The first version of PICSearch is able to retrieve images from a local database. Since there are a lot of image sources on the Internet, the obvious and desired extension of the system is to make the queries work over the Internet. We have developed a client-server extension to the PICSearch system, which we refer in the sequel by using name NetPICSearch. The usage of NetPICSearch has been made as easy as possible. Both the (local) server and the client can be accessed through the same interface. The client executes queries by forwarding the requests of the user to the connected server. Thus, the database accessing done by the server is completely hidden from the client.

From the efficiency point of the view, the crucial decisions to be done in a client-server architecture are 1) the distribution of work across the client and server and 2) communication between the two ends. In our system the server plays a very important role; almost all the important matters concerning the retrieval are actualized by it. We use different techiniques to minimize the communication rate, e.g. in order to get results of a query as fast as possible only thumbnails of matched images are retrieved.

The organization of this paper is as follows: In the following section we shortly refer to the most similar projects to ours. In section 3 we give an overview to our system. Section 4 gives some details of the client-server architecture and section 5 of the communication protocol. In section 6 a procedure for embedding an algorithm in our system is given and eventually, we conclude the paper with some discussion in Section 7.

\footnotetext{
*The financial support of the Helsinki Graduate School of Computer Science and Engineering is appreciated.
} 


\section{Related Work}

The most known image retrieval systems are $Q B I C$ and Virage. They are commercial products that enable the addition of some custom-defined components. The QBIC system [2, 3] has been developed at IBM. It offers several query options: query by colour, by example, by sketch, by texture or on the position of the objects that the query image contains. These attributes can be combined into a multi-feature query.

The Virage Engine [1] is based on the VIMSYS model [4]. Image features considered in Virage include texture, colour and structure which are extracted by different processes. Every computational process is assigned with a corresponding distance metrics which can be adjusted by the user. Virage technology has been combined with an object relational database called Illustra.

Orphanoudakis, Chronaki and Kostomanolakis have developed a system called $I^{2} \mathrm{C}$ for medical image retrieval. In their system the user can embed new description types, segmentation algorithms, image processing algorithms and properties. Due to the rather dissimilar application domain they provide some original services, e.g. they provide a classification-tree to browse/edit. Different attributes can be assigned to the different classes.

Van den Berg et al. [11] have concentrated on integrating image processing and database paradigms. Like us, they try to facilitate the construction of an image retrieval system. However, their approach is rather different: As we have concentrated on thorough definition on the database interface (and provide only straightforward database implementation) they have integrated a certain object-oriented database (based on MONET database) with their detector based image processing module.

Furthermore, in [9] Mills, Moody and Rodden describe a challenging approach to information retrieval by presenting high-level abstraction for IR systems. Their system, Cobra, is intended to handle multimedia collections, including a combined text and audio collection, and an image database. So far their system supports full-text querying (including phrases) and the first experiments have been promising. They are going to continue the development of the system in order to support other multimedia collections.

\section{What is PICSearch}

PICSearch is a platform system that is designed for embedding content-based image retrieval algorithms by using provided templates. It is implemented by students having their software program course. We have tried to keep the system as small and simple as possible, with well-defined module and program interfaces in order to provide a system that is extendible, portable and easy to learn.

PICSearch is portable. It is programmed in $\mathrm{C}++$ and follows the principles of object-orientation. The user interface was constructed with script language Tcl/Tk. By using these tools and restricting operating-system dependent code into as small areas as possible, we were able to make PICSearch an easily portable system.

PICSearch is extendible. The embedding of image retrieval algorithms is tried to facilitate by providing templates. However, adding new retrieval algorithms is not the only way to extend PICSearch. Image processing tools and an existing image database engine can also be integrated to the system.

The most essential limitation for embedding an algorithm in the PICSearch system is that the algorithm has to support the idea of something that we call a signature, i.e. a sequence of some values. The length of these sequences is not limited, and the interpretation of a particular signature has to be coded by the algorithm developer. The signature is computed out of every image and it is to be used in comparison to determine the similarity of two images.

Since the primary aim of our project has been to provide a platform for researchers, only a small set of algorithms is provided at the moment: searching by $i d$ (picture identifier), by associated keywords, by average colour and a Waveletbased matching algorithm by Jacobs, Finkelstein and Salesin [5]. By providing these algorithms we were able to test the system and assure ourselves that it works correctly.

PICSearch is freely available. It is distributed under the GNU General Public License.

\section{Architecture of NetPICSearch}

NetPICSearch is divided into a server and a client application. The server deals with multiple clients simultaneously. 


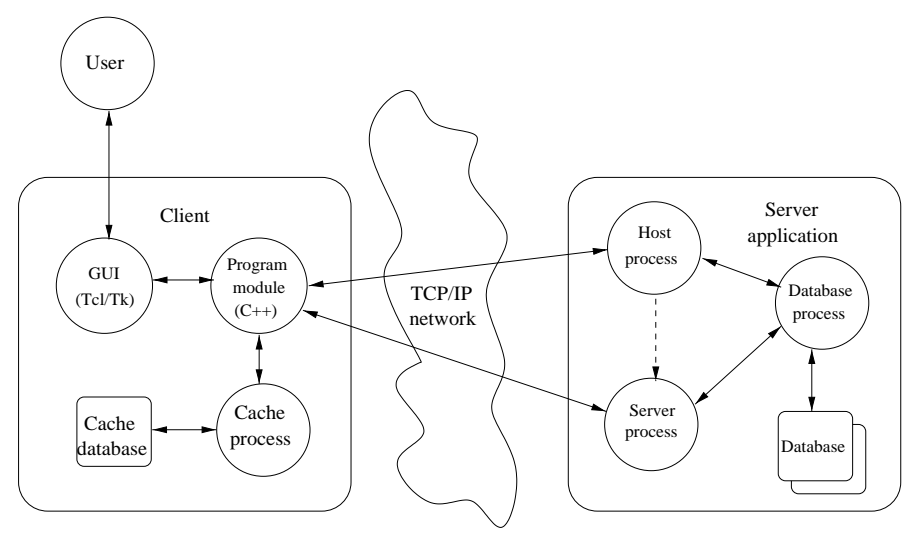

Figure 1: The main architecture of the system.

Each client, on the other hand, may be connected to one server at a time. The network protocol used in communication between the two applications is TCP/IP. The main architecture is illustrated in Fig. 1.

The client provides a graphical user interface (See Fig. 2) for normal retrieval use as well as for the system administrators. Users are identified by a normal login/password procedure. The client also provides a cache to temporarily store result images. After a query has been executed by the server a list of matching pictures is returned to the client. The client uses this information to do a lookup from the cache for identical thumbnails. Only the thumbnails which are not found in the cache are requested from the server and upon retrieval stored in the cache for later lookups.

The server is a background process without a separate user interface. The server administration, i.e., user management and database maintenance may be done remotely (when the client and the server are running in separate machines) over the network. Server administrators can create and delete repositories and specify different login names to give access to the images. Server installations may offer multiple image collections for the client applications. Each collection may contain a number of images and have user rights defined separately. Thus, public collections as well as more or less restricted collections can be created.

\subsection{Client}

The client application consist of a graphical user interface, a program module and a cache. The client program module is programmed in C++, whereas the graphical user interface (GUI) is made up of Tcl/Tk scripts. The architecture and the functionality of the client program are almost the same as in the first version of the PICSearch. Only some minor changes and additions were necessary because of the network support and the user management.

Because the server part of the NetPICSearch was designed to serve many different clients concurrently all around the network it was reasonable to add simple user management in order to control access to the server part. The server options and the user management information are stored to the data structures of the client program module. If the client has superuser rights of the server, it receives this information from the server after the connection is established. The information is stored both to the server and the client in order to minimize the network traffic between them, because it is very likely that a superuser updates several administration information at a time. After the information has changed the data structures of both the client and the server are updated.

The GUI module accesses the program module through a well-defined set of functions, one function for each action the user may perform. As the program module proceeds it can either give the possible results back to the GUI immediately, if it is able to complete the given task itself, or it can forward a request to the server and wait for its reply, after which the possible results from the server are given to the GUI.

Because the data structures and the functionality of the client program are mostly located in the program module, the client can be easily separated from the Tcl/Tk and replaced by another GUI mechanism. 


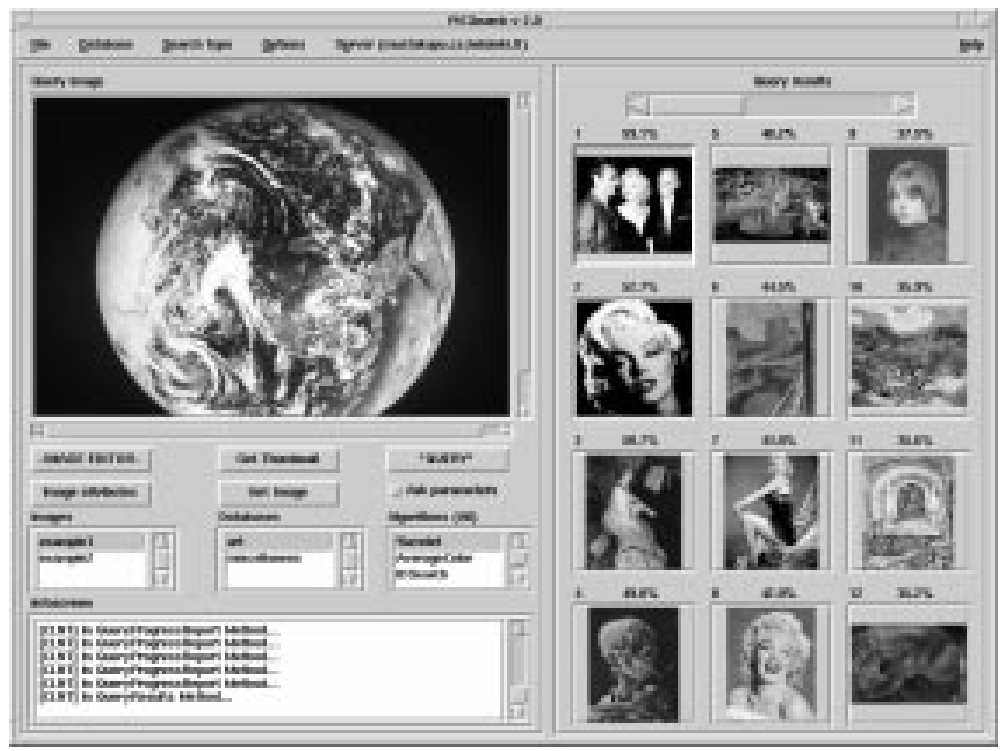

Figure 2: The GUI. A query has been executed (the query image does not exist in the database).

\subsection{Server}

The server application consists of several processes that are all programmed in $\mathrm{C}++$. Each different process is encapsulated into a separate class. There are three kinds of processes in the server application: a host process, a database process and a varying amount of server processes. The relations between the processes are illustrated in Fig. 1.

The initial process in the server application becomes the host process. The database process is created during the host initialization sequence. Also the communication channels between the host and the database processes are set up.

The host process listens to a predefined TCP port for connection requests sent by client applications. Upon receiving a request the host process checks for adequate user authorization before starting a dedicated server process for the client. Further communication from the client application is handled by this dedicated server process on a separate TCP port.

The first of the two authorization checks is done by the host process when a client tries to connect to the server application. The host is responsible for letting only a predefined number of authorized users to get service. There are two levels of authorization on the host level: access and superuser. Only one superuser is allowed to be logged on at a time. This is due to the fact that a part of the server application's data structures are cached on the client application side. The superuser can log in even though the maximum number of non-superusers are already logged in.

Each server process communicates with one client application. Its task is to receive, interpret and execute requests from the client. Most of the requests need sufficient authorization in order to be executed. When the client sends a query request and an image the server utilizes the database process to produce a set of matched images. Finally the results are packed into a compact form and sent to the client application.

If the server process is idle (i.e. no requests are received from the client application and no request is currently being processed) for a predefined period of time it will close the network connection and die. This is needed because the number of server processes is limited by the host process for heavy traffic control.

Both the host and the server processes use the same database process and the same communication channels to access the stored information. The communication between the database and the other processes is done via two pipes. Every process that uses the database process uses the same pipe pair to send the database operation for the database process and to receive the results of the operation. The usage of the pipes has to be serialized to prevent the messages from interfering with each other. This is achieved by using a semaphore.

Each database operation starts by acquiring the semaphore for the communication pipes. This provides exclusive access to the database. Once the semaphore is acquired, the database operation is sent to the database process. After executing the operation the database process sends the query result to the query result pipe that is listened by the 
operation sender. After reading the result the semaphore is released to allow the next process to use the database.

One table in the NetPICSearch repository consists of an index file and a datafile. The index file speeds up the data file access. The database process has control over multiple server databases. The server database contains information about the image databases, users and server settings. An image database contains collection of images and their parameters, signatures, and smaller thumbnail presentations of the images.

The Server Protection. The server application is protected against possible hostile actions from the network. Any connection attempt to the server must follow a precise predefined form or the connection will be rejected by the server application. At the very beginning of the connection the client's authorization is checked by the server application and if the check fails the connection will also be rejected. Most of the operations offered by the server application are available to superuser level clients only.

Only data sent to the server application in a proper form is accepted and processed. Thus only the functions specified in the client/server interface can be performed and they do not contain any potentially dangerous operations to the system the server application is running on.

One important factor increasing the system's security is the fact that the passwords are never transmitted from the server application to the client in any case. The client application is built in such a way that it can operate without the knowledge of the passwords even when accessing the server in superuser level. All password verification is done by the server application.

NetPICSearch network connections can be controlled externally by a firewall similarly as any other network application using the TCP protocol. Firewalls can therefore present a limitation to the network accessing of NetPICSearch. The TCP port which is used by the server application's host process is configurable in the sources, though.

\section{The Communication between the Client and the Server}

NetPICSearch can be used in any TCP/IP network including the Internet. Due to the time limitation it is implemented synchronously. A NetPICSearch server communicates only with a NetPICSearch client. At connection time the server may advertise itself by providing a HTTP address that is to be used by the client (if the client has a WWW browser). Otherwise, the communication between the two consists solely of messages needed to run image queries.

Because the key functionality of the system is located in the server program a significant amount of network traffic between the client and the server is necessary. In a low bandwith network this could slow down the system considerably. To avoid that, we have concentrated on using as short messages as possible.

In our original implementation the client computed the signatures out of the query images and sent them to the server for comparison with the signatures stored in the repository. Though in many cases the network traffic was minimized this implementation caused problems in those situations where the two ends had different versions of the same algorithms or two totally different algorithms had the same name.

Because of those problems we have a new implementation with a central algorithm repository. The server was chosen to determine which algorithms are available. The drawback of this change is the momentary increase of the network traffic because the full size query image has to be transferred from the client to the server in order to get the signature computed out of it.

Since we use preprocessing of the database images and we want to minimize the messages (and therefore we do not want to send an algorithm from client to server), only algorithms combined to the connected database of a server can be used. If we had enabled the sending of algorithms, the increased communication would not have been the only reason to slow down the retrieval, but also a remarkable time would have been spent on on-line computation of the signatures out of database images. When the server has executed the query, it sends only the thumbnails of the matching images to the client, not the actual full-sized versions of the images.

When the user has received the images provided by a query, he may execute a query with one of the result images as the new query image. In this case the server does not need the query image since the image already resides on the server. If the user has found an image that meets their demands, it can be transferred from the server just by clicking a button on the screen. After receiving the whole image, he may connect to another server and start a new series of queries with 
this image. Thus, the querying process can be a long chain of queries where different servers and databases all over the Internet can be used to achieve a satisfactory result.

\section{Embedding Algorithms}

In this section we give a short example of embedding a simple matching algorithm based on the average colour of images. For a more complex algorithm the procedure is analogous, but the computation is more involved.

Embedding a new algorithm in our system requires little knowledge about the inner workings of the system. Only two files under new names have to be copied and a non-trivial code for six functions has to filled in. The essential parts of the $\mathrm{C}++$ code can be seen in Appendix 1.

1. The signature was designed to be fixed-length ( 3 bytes), thus one variable for the average value of each colour component were added to the Signature-class implementation.

2. Writing and reading these values to/in a memory buffer was trivial (functions getBin and setBin). Generating a human-readable representation of the signature was equally trivial.

3. The algorithm was designed to use three parameters, each controlling whether to use a corresponding colour component during a query. Hence one variable for each parameter was added to the Algorithm-class implementation. Straightforward code for reading/writing the parameters was added (functions getParams and setParams).

4. A new window was created (requiring 15 lines of Tcl/Tk-code) in the GUI in order to control the parameters.

5. The actual computation of the signature was coded: a simple loop summing up the colour component values from a given memory buffer and finally dividing them by the size of the image and setting the values into a new Signature-class instance.

6. Finally, the method for comparing two signatures was coded to interpret the colour components as threedimensional co-ordinates. The distance between two given signatures is calculated out of this representation and the solution is scaled to the required interval (0-100).

To test the algorithm, the system was recompiled and the database automatically processed through using the new algorithm. The whole process, the implementation and the recompilation, took about two hours.

\section{Discussion and Future Work}

We have presented a client-server extension to the PICSearch system, which helps researchers test their algorithms. The user of NetPICSearch programs the algorithm in th C++ language using our templates, defines certain control parameters and codes the method for comparing two images (or signatures). As a result, they can test their algorithms on different image sets residing in different NetPICSearch servers without the bother of creating a whole retrieval system.

Though, the PICSearch system was originally designed to researchers the "end users" are not forgotten in our system. The graphical user interface has been found out to be clear and easy-to-use by potential end users. The system has been implemented by student groups having their software project course. As they have found out the software is a useful tool also for themselves their have gone into a detailed design and implementation, resulting to a useful piece of software.

The contribution of PICSearch version 1 was that it is freely distributed, extendible and portable. These targets are to be kept in our minds in the forthcoming development of the system. However, for the present we do not have a $\mathrm{C}++$ compiler for Windows environment, which has enforced us to leave the testing of NetPICSearch in this particular environment in the future.

In a client-server tool the distribution of the work between the client and the server, and the communication rate play a very important role. In our system almost all the important functionality is included in the server. The most important role of the client is to forward the requests of the user to the server, and present the query results to the user. The communication rate is usually the "bottleneck" of the system. Therefore, we have paid attention to the design of the communication protocol; as little data is sent to the network as is possible. Furthermore, another very important property is a good server protection. Should a client-server system to be widely used, it needs to be protected against possible hostile actions from the network. We have given an overview of the server protection in our system. 
The development of PICSearch is to be continued. Currently we are struggling up to automatize the embedding procedure. Further improvements could be e.g. the possibility of combining different retrieval algorithms. However, this can be rather difficult because of the diversity of the retrieval algorithms; the model has to support any combination of the algorithms by knowing almost anything beforehand about them.

Also systematic experiments on our synchronous data transfer has to be done. The drawback of it is that if the network is temporarily very slow for some reason, the GUI "freezes". This is because the execution stays in the client program module while it is waiting for the answer. If the experiences are going to be too bad we will displace it by asynchronous transfer.

Furthermore, the development and implementation of an efficient indexing structure is left over for future projects.

Everything associated with PICSearch can be found at: http://www.cs.Helsinki.f//Klemstro/picsearch.html. If you consider the system useful or have some comment on it, please let us know (e-mail: picsearch-list@ cs.helsinki.fi).

\section{Acknowledgements}

The following persons have been participating in the project: Petri Kanerva, Anita Kasari, Samuli Kaski, Sami Koponen, Jouni Korte, Timo Koskinen, Pyry Kuusi, Pasi Kyheröinen, Jani Leinonen, Sari Männynsalo, Mika Pennanen, Pekka Päiväkumpu, Juha Riihimäki, Janne Teinilä, and Timo Virtanen. Their assistance is appreciated.

The comments of professor Esko Ukkonen and professor Tapio Takala are also appreciated.

\section{References}

[1] Bach JR, Fuller C, Gupta A, et al: The Virage image search engine: An open framework for image management. In: Proceedings of the SPIE: Storage and retrieval for still image and video databases IV. 1996

[2] Faloutsos C, Barber R, Flickner M, et al. Efficient and effective querying by image content. Journal of Intelligent Information Systems 1994; 3: 1-28

[3] Flickner M, Sawhney H, Niblack W, et al. Query by image and video content: The QBIC system. IEEE Computer 1995; 28 (9): 23-32

[4] Gupta A, Weymouth T, Jain R: Semantic queries with pictures. In: Proceedings of the 17th international conference of VLDB. 1991, pp 69-79

[5] Jacobs CE, Finkelstein A, Salesin DH: Fast multiresolution image querying. In: SIGGRAPH'95. 1995, pp 277-286

[6] Lemström K, Korte J, Kuusi P, Kyheröinen P, Päiväkumpu P: PICSearch - a platform for image content-based searching algorithms. In: Proceedings of the sixth international conference in Central Europe on computer graphics and visualization (WSCG'98). 1998, pp 222-229

[7] De Marsicoi M, Cinque L, Levialdi S. Indexing pictorial documents by their content: a survey of current techniques. Image and Vision Computing 1997; 15: 119-141

[8] Mehrotra S, Rui Y, Ortega M, Huang TS: Supporting content-based queries over images in MARS. In: Proceedings of the IEEE international conference on multimedia computing and systems ICMCS'97. 1997

[9] Mills T, Moody K, Rodden K: Cobra: a new approach to IR system design. In: Proceedings of RIAO'97 (Recherche d'Information Assistée par Ordinateur). 1997, pp 425-449

[10] Orphanoudakis SC, Chronaki C, Kostomanolakis S. A system for the indexing, storage, and retrieval of medical images by content. Journal of Medical Information 1994; 19 (2): 109-122

[11] van den Berg CA, van den Boomgaard R, Worring M, Koelma D, Smeulders A: Horus: Integration of image processing and database paradigms. In: Proceedings of the first international workshop of image databases and multi-media search. 1996, pp 226-234 


\section{Appendix 1 The class skeletons for embedding an algorithm}

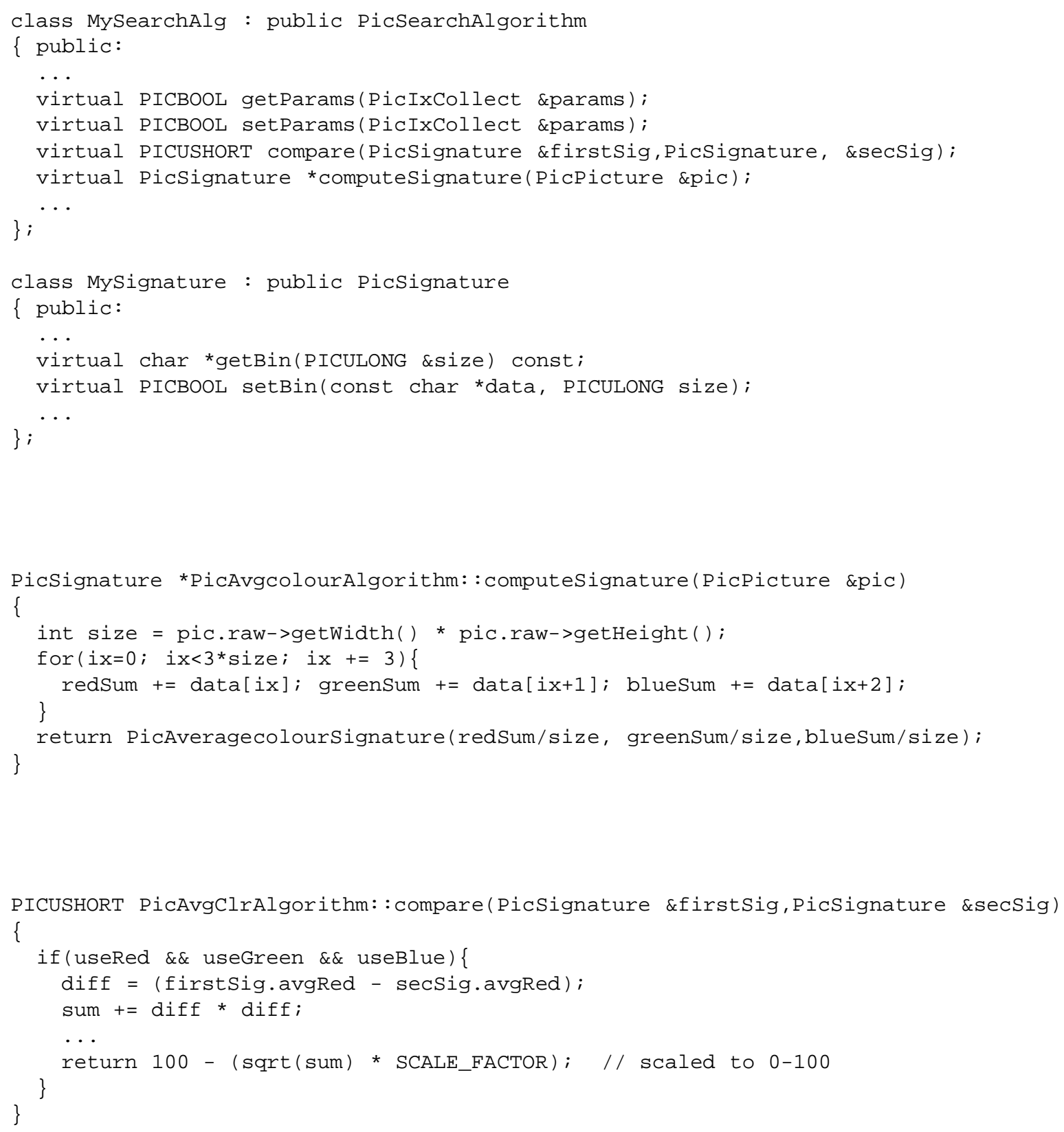

